\title{
Model-free adaptive speed control on travelling wave ultrasonic motor
}

\author{
Sisi Di, Huafeng $\mathrm{Li}^{*}$
}

\begin{abstract}
This paper introduced a new data-driven control (DDC) method for the speed control of ultrasonic motor (USM). The model-free adaptive control (MFAC) strategy was presented in terms of its principles, algorithms, and parameter selection. To verify the efficiency of the proposed method, a speed-frequency-time model, which contained all the measurable nonlinearity and uncertainties based on experimental data was established for simulation to mimic the USM operation system. Furthermore, the model was identified using particle swarm optimization (PSO) method. Then, the control of the simulated system using MFAC was evaluated under different expectations in terms of overshoot, rise time and steady-state error. Finally, the MFAC results were compared with that of proportion iteration differentiation (PID) to demonstrate its advantages in controlling general random system.
\end{abstract}

K e y w or d s: data driven control, model free control, speed control, ultrasonic motor

\section{Introduction}

Speed and/or position control of ultrasonic motors is a crucial issue in engineering sector due to its theoretical challenges and special applications. Travelling ultrasonic motor, which typically adopts a bonded structure and works on temperature-sensitive piezoelectric materials, is prone to be disturbed by various interferences especially the internal and external temperature. Thus, the nonlinearity caused by the piezoelectric component and its interaction with the nearby layers, namely, the friction layer, stator and rotor, poses a challenge to the modeling and controlling of the system. Two popular ideas of USM control are the PID type control technique based on the classical PID rules and the applications of artificial neural network (NN) technique in adaptive control developed in recent years.

In the early stage, researchers have made much effort on speed and/or position control via PID and NNs, which was usually combined with fuzzy logic strategy. Take the University of Ryukyus as an example, [1] applied the conventional PID control method on the USM system to obtain the desirable speed and/or position by controlling three features of the driving signal, namely, the voltage, phase, and frequency. As comparison showed the advantages of adaptive controller over PID in [2], they began combining PI/PID controller with other state-of-art theories at that time, such as the repetitive compensator for the speed ripple caused by temperature rising [3] and the adaptive controller for online parameter identification [4].

Besides, to enhance the tracking and control performance of his former model-following error-driven fuzzy adaptive mechanism, [5] implemented a three-layer online trained NN controller with variable learning rates to enhance the transient response and to increase the robustness of the USM drive system [6]. The NN was designed to tune the output scaling factor of the fuzzy controller. To discover more sensitivity information of the drive system, [7] separated the fuzzy neural network $(\mathrm{FNN})$ scheme into two parts, the FNN identifier and FNN controller. However, more information claimed larger calculation. Then, [8] introduced a delta adaptation law into the backpropagation algorithm used to train the $\mathrm{NN}$ to avoid heavy computation and to increase the online learning rate of the weights. Later on, thanks to the deeper study of the internal principles of USM and various models (mechanical-electrical model, electrical-equivalent model, fiction model, etc) of its working process, researchers preferred to control the speed of travelling rotating ultrasonic motor (TRUM) and/or position based on these models for higher accuracy. For example, Shenglin Mu processed a PID type internal model control (PID-IMC) using NN. The NN controller combined with particle swarm optimization (PSO) based on probability is employed for tuning the PID gains in the PID-IMC for compensating the characteristic changes and non-linearity in USM. Meanwhile, the NN was learned by updating its weights online using PSO algorithm [9-11], [12] and [13] turned to the pole-assignment strategy for proper PID parameters selecting of a system identified from step response and the expected closed-loop characteristic. Hammerstein model was also identified using PSO for its advantages over simple difference equations in depicting the characteristics of nonlinear systems [14]. Then, the self-tuning nonlinear generalized predictive speed control strategy of

* State Key Laboratory of Mechanics and Control of Mechanical Structures, Nanjing University of Aeronautics and Astronautics, Jiangsu 210016, China, michelledss@sina.com,lihuaf@nuaa.edu.cn 


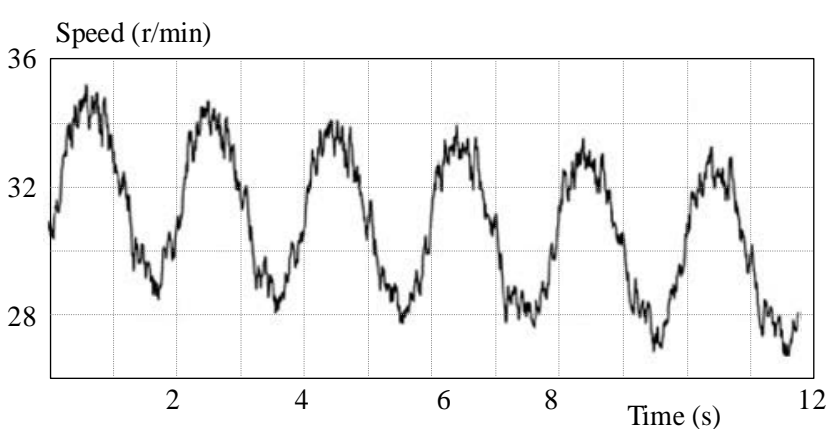

Fig. 1. USM speed in respect to time at $41.9 \mathrm{kHz}$

an ultrasonic motor was realized based on this Hammerstein model [15]. [16] proposed a simpler intelligent PID speed control strategy of TWUM involving only two expert rules to adjust the PID control parameters based on the current status which allowed less calculation and cheaper MCUs.

Present, considering the greater capacity of digital technique for processing large amount of data and growing demand for fast response in applications, the concept of DDC is called up especially for nonlinear coupling system, such as USM. When the system contains more unpredictable nonlinearity that is hard to reach an analytical solution, it is reasonable to expect an adaptive control system independent of the system model which is only based on information from the I/O data of the controlled plant. DDC, including the popular strategy of PID, reaches the goals depending only on the control inputs and feedback outputs except the reluctant model functions.

In this paper, the model-free adaptive control(MFAC) proposed by Zhongsheng Hou in [17] was implemented in the speed control system of USM. This paper extent the state of the art, where model-based controlling method fails to timely track the instant response of the state changing due to the complex calculation of the nonlinear model parameters from large matrix or equations. The comparison with traditional PID controller illustrated its great potential in the online control with regard to its fast response and robust self-adaption.

\section{Problem}

Although the proposed method is independent on the system model, an experiment-based model mimicking the USM working system has to be introduced for simulation to verify the feasibility of MFAC. The model discussed in this section, which contains the measurable nonlinearity and uncertainty, is established for simulation only other than the future application of MFAC on controlling.

The interaction between the speed of USM and its excited signal, surrounding environment, and other elements is time-varying and high-nonlinear. Empirically, the frequency of the excited voltage signal is often the priority in choosing the controlling object thanks to a wider control range than that of the voltage-based strategy. Also, it is more feasible to control the frequency than the phase while the phase-based method would leads to a zero-zone problem. Besides, the speed decreases as time goes by when the resonant frequency is changed by the increasing temperature. Thus, a control-friendly and experiment-based model in terms of the operating time and frequency of the excited voltage was considered to describe the ultrasonic motor control system.

\subsection{Experiment setup}

To obtain the parameters of the system, a whole setup (Fig. 1) was established to measure the velocity of USM varying with frequency and time. When the direct digital synthesizer (DDS) was programmed to generate the highfrequency periodic pulse for sine wave signal, the amplifier contributed to a pair of sinusoidal voltage signal with a phase difference of $90^{\circ}$ which was transformed from the digital pulse using the $\mathrm{D} / \mathrm{A}$ processor packed into the DDS module. To obtain the relation between the speed and frequency, and speed and time respectively, the amplitude and initial phase were kept at $360 \mathrm{Vpp}$ (peakto-peak voltage value) and $0^{\circ}$ respectively. The TRUM60 USM (Fengke Co., Jiangsu, China) was selected for all the experiments. To obtain the speed value of the motor, the intervals between neighbor pulses emitted from the incremental rotary encoder $(N A I R C$, China) was counted in the programmable-MCU PSoC 5 (Cypress, USA) and sent out through a serial port for the following process. The Instrument Control Toolbox of Matlab was used to receive the serial data for the final calculation of speed considering the resolution of the encoder.

To obtain the relation between the speed and frequency, the speed at different frequencies from $40.5 \mathrm{kHz}$ to $43.0 \mathrm{kHz}$ were measured with the benchmark.

However, for the speed-time relationship, the range was cut into a smaller range. Practically, USM usually operates at a frequency a little higher than the resonant frequency avoiding the damage on the piezoelectric ceramic brought by the sympathetic vibration. In the case of TRUM-60, the allowed working frequency should be no less than $41.9 \mathrm{kHz}$. On the other hand, the speed value at a frequency above $43.0 \mathrm{kHz}$ is usually less than $5 \mathrm{r} / \mathrm{min}$ indicating a small slope of speed in respect to time. Thus, the change of slope in respect to frequency contributed little to the identification of the speed-time model when the USM worked at a voltage higher than $43.0 \mathrm{kHz}$. In this case, speed data varying with time at frequencies from $41.9 \mathrm{kHz}$ to $43.0 \mathrm{kHz}$ were measured with the benchmark. Finally, 12 groups of data, each of which was tested under the frequency $0.1 \mathrm{kHz}$ larger than that of the former one, were collected for the following analysis.

\subsection{Model presented}

All the 12 groups of data were drawn with Matlab to demonstrate the speed varying with frequency and time. Since all the curves enjoyed a similar shape except for different amplitudes, any figure of these alone is enough 


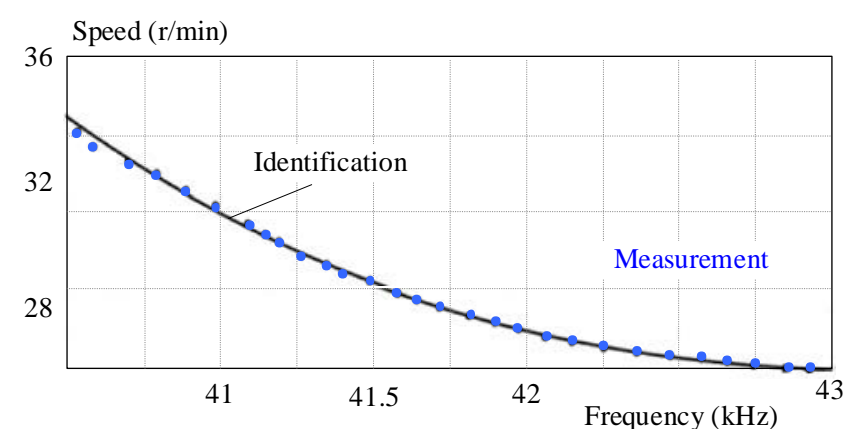

Fig. 2. Measurements and identification results of speed in respect to frequency of the excited signal

Table 1. The search range and boundary wall defined of some parameters

\begin{tabular}{ccc}
\hline Particle name & Search range & Boundary wall \\
\hline$\tau$ & $(0, \infty)$ & $\tau=1$ \\
$\omega$ & $(0, \infty)$ & $\omega=1$ \\
$\varphi$ & $(-\pi, \pi)$ & $\varphi_{\min }=-\pi, \varphi_{\max }=\pi$ \\
\hline
\end{tabular}

for a fully explanation of the speed-time model. In this paper, the figure of $41.9 \mathrm{kHz}$ was taken as an example.

As shown in Fig. 1, the curve of the open-loop velocity shared the shape with a sinusoidal wave. Considering the speed's final convergence to zero, the following basic function was referred to

$$
y=A e^{\frac{t}{t_{0}}} \sin (\omega t-\varphi)
$$

where $e^{\left(t / t_{0}\right)}$ indicates a decreasing amplitude of a sine function. Theoretically, (1) has been proved to be converging to. Furthermore, Fig. 1 showed a gradual damping peak value and the curve is symmetrical about an exponent-like line. In this case, (1) is modified for the identification of system shown in Fig. 1 and (2) was introduced to express the experiment-based practical speedtime model of USM

$$
v_{t}=V_{f} e^{\frac{t-t_{0}}{t}}(1+k \sin (\omega t-\varphi))
$$

where $v_{t}$ is the transient speed varying with the time, $V_{f}$ is the starting steady speed in respect to frequency.

\section{Identification of proposed model}

According to the mechanism of USM, $V_{f}$ varies with the frequency of the excited voltage whose rules can be figured out using a simple polynomial function. The other unknown factors presented in (2) have to be identified and PSO was introduced for the estimation.

\section{$3.1 V_{f}$ identification}

A three-order polynomial was adopted to describe the speed in respect to frequency based on the measurements obtained in Section 2.1

$$
V_{f}=a f^{3}+b f^{2}+c f+d
$$

where $f$ is the frequency of the excited voltage signal, and $a, b, c$ and $d$ are the coefficients of the polynomial to be identified. The frequency and speed data were scaling for well conditioning.

The "polyfit" function in Matlab was applied for a quick solution to the equation and came up with a $4^{\text {th }}$ vector $[a, b, c, d]$ as the result. Substitute the solution vector into (3), we have (4) as our final speed-frequency model for estimation of $V_{f}$

$$
\begin{aligned}
V_{f}^{\prime}= & -2095.85 f^{\prime 3}+6992.43 f^{\prime 2}-7770.46 f^{\prime} \\
& +2876.29857
\end{aligned}
$$

where $V_{f}^{\prime}$ and $f^{\prime}$ are the scaling values of $V_{f}$ and $f$ respectively. The identification results agreed well with the measurements in Fig. 2.

\subsection{PSO identification}

The particle swarm optimization realized by the iterations of updating each particle's personal best (pbest) and the global best (gbest) to "pull" the swarm of particles to the supposed best position. Each particle in the solution space moves towards the gbest position at the velocity calculated from

$$
\begin{aligned}
v_{n}^{k+1}=l \times v_{n}^{k}+c_{1} \times & \operatorname{rand}() \times\left(\text { pbest }_{n}-x_{n}^{k}\right) \\
& +c_{2} \times \operatorname{rand}() \times\left(\text { gbest }_{n}-x_{n}^{k}\right) .
\end{aligned}
$$

where $v_{n}^{k}$ and $x_{n}^{k}$ are one particle velocity and coordinate in the $n$th dimension after $k$ th iteration respectively, pbest $_{n}$ and gbest $_{n}$ are the best location of the particle and swarm in the $n$th dimension respectively, and $l, c_{1}$, and $c_{2}$ are self-defined numbers for the algorithm.

Then the new position is updated as

$$
x_{n}^{k+1}=x_{n}^{k}+v_{n}^{k+1} .
$$

\subsubsection{Solution space establishment}

Referring to (2), a five-dimensional solution space is introduced for the identification of five unknown factors, $t_{0}, \tau, k, \omega$, and $\varphi$. According to the practical range of each parameter, reasonable specification of the minimum and maximum value is given in Table 1 .

Since an exponential function is convex when $\tau$ is larger than zero, which agrees with the damping tendency of the measurements, $\tau$ is limited within the positive half of the axis. The oscillation frequency $\omega$ must be above zero, considering its physical significance in practice. A range within $-\pi$ to $\pi$ is enough for the identification of initial phase of the oscillation $\varphi$ due to the sinusoid's periodic characteristic. 


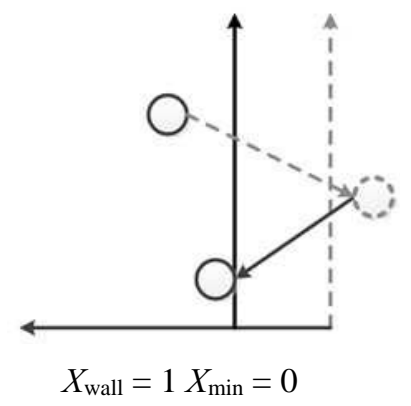

Fig. 3. Absorbing-partial-reflecting walls

Table 2. Identification results of PSO

\begin{tabular}{lccccc}
\hline No. & $f(\mathrm{kHz})$ & $\tau$ & $k$ & $\omega$ & $\varphi$ \\
\hline 1 & 41.9 & 46.283 & 0.085 & 10.891 & 0.785 \\
2 & 42 & 25.271 & 0.082 & 10.91 & -0.471 \\
3 & 42.1 & 23.877 & 0.087 & 10.941 & -1.212 \\
4 & 42.2 & 18.648 & 0.089 & 10.99 & -2.534 \\
5 & 42.3 & 15.591 & 0.088 & 10.977 & -0.863 \\
6 & 42.4 & 13.926 & 0.093 & 10.98 & 0.737 \\
7 & 42.5 & 13.771 & 0.089 & 11.078 & -1.569 \\
8 & 42.6 & 18.106 & 0.09 & 10.91 & 2.173 \\
9 & 42.7 & 21.976 & 0.086 & 10.918 & -2.715 \\
10 & 42.8 & 20.285 & 0.092 & 10.988 & -1.487 \\
11 & 42.9 & 16.171 & 0.084 & 10.985 & -2.086 \\
12 & 43 & 28.241 & 0.09 & 10.861 & 2.513 \\
\hline
\end{tabular}

\subsubsection{Boundary limitation}

The "absorbing-partial-reflecting walls" were imposed to confine the particles within the solution space. When a particle hits the boundary of the solution space in one of the dimensions, the coordinate of the particle in this dimension is reset to be a predefined value within the solution space. To avoid zero denominators, the walls of $\tau$ and $\omega$ were built at a position slightly larger than zero, namely, "partial-reflecting". The adjustment process is drawn in Fig. 3 [18].

\subsubsection{Selection of parameters}

First of all, to reach a compromise between the efficiency and reliability, a population size of 30 was adopted in this paper. Then, an inertial weight $\omega$ linearly decreasing from 0.9 to 0.4 during the iterations was employed to achieve the balance exploration and exploitation [19]. Also, the developers of PSO suggested a constant of 2 for both $c_{1}$ and $c_{2}$ in order to give the stochastic factor $\operatorname{rand}()$ a mean of 1 , so that particles would "overfly" the target about half the time [20].

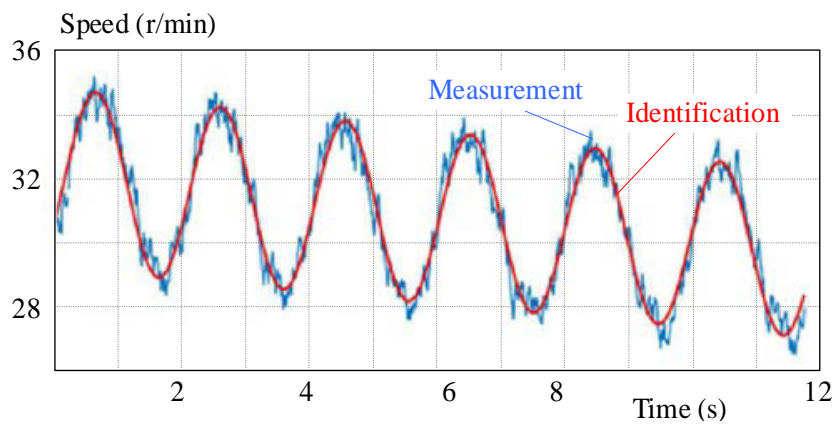

Fig. 4. Identification results when USM working under a voltage of $41.9 \mathrm{kHz}$

\subsubsection{Application on USM}

According to the above analysis, the calculation was carried out using Matlab 2015b. Since all the 12 groups of data share the same shape, the situation when the USM works under the voltage of $41.9 \mathrm{kHz}$ was taken as an example (Fig. 4) to avoid repetition of illustrations.

All the 12 groups of data were imported to identify speed-time relationship under different frequency and the PSO identification results are represented in Table 2. It came up with a random value of $t_{0} \in(-2,2)$ for all the groups, same $\tau$ and $\varphi$ for a certain group but quite different among all the 12 groups which indicated that they were irrelevant with the frequency. So the value of $t_{0} \in(-2,2), \varphi \in(-\pi, \pi)$ and $\tau$ were randomly selected for a rough simulation of the speed-time model of USM control system. Furthermore, similar $\omega$ and $k$ were observed among all the groups, so the mean of $\omega=10.952$ and $k=0.088$ were determined as the final solutions. In this way, the experiment-based speed-frequency-time model was finally determined when combine the identification results of (2) with (4). It described the timevarying nonlinearity and uncertainties which often occur in real USM system.

\section{The development of MFAC}

\subsection{Overview of MFAC}

The MFAC is developed based on the following discrete SISO nonlinear systems

$$
\begin{aligned}
& y(k+1)= \\
& =f\left(y(k), \ldots, y\left(k-n_{y}\right), u(k), \ldots, u\left(k-n_{u}\right)\right)
\end{aligned}
$$

where $u(k), y(k) \in R$ are the input and output value at $k$ time respectively, $n_{y}$ and $n_{u}$ are the orders of the input and output respectively, $f(\ldots)$ is a general nonlinear function. The efficiency of (7) in representing a broad range of nonlinear behavior has been proved in subsequent publications since it was first introduced as a NARMAX model, which stands for nonlinear autoregressive moving average exogenous inputs, in 1981 by Stephen A. Billings [21]. 
When a linearized NARMAX model encapsulates all of the unknown parametric dynamics into a single timevarying nonlinear parameter of the system states and virtual control signals, the computational complexity of the proposed algorithm is modest, enabling its implementation in a real-time system even when considering long prediction horizons. Then, popular linear estimation and optimization approaches could be applied on the pseudolinear system for the online controlling with only closedloop operating data required rather than the details of the model information.

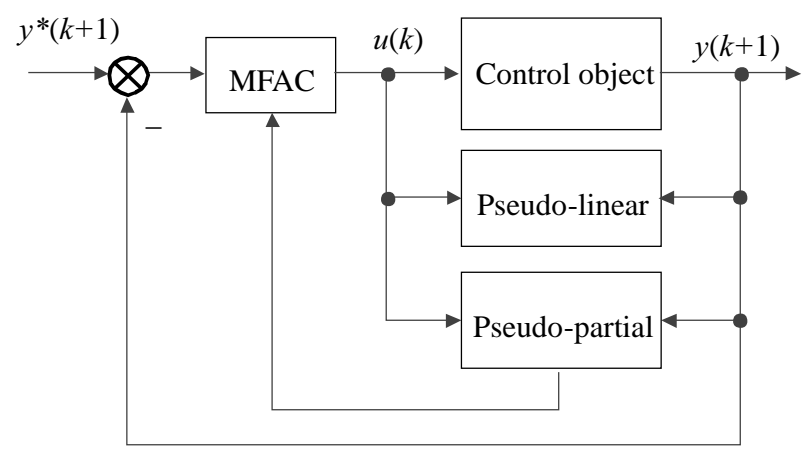

Fig. 5. The overall flowchart of MFAC-based control scheme

The MFAC method can be classified into three branches in terms of how the I/O data are processed for the linearization of NARMAX model, that is: the pseudo partial derivative (PPD), pseudo gradient (PG) and pseudo Jacobian matrix (PJM). In this paper, PPD is first attempted for the controlling of USM. According to the close-loop control strategy of MFAC presented in Fig. 5 the proposed method contributes to a proper input $\mathrm{u}(\mathrm{k})$ based on the PPD estimated for the pseudo-linear model from the I/O data and helps the system with an ideal output $y(k+1)$ based on the control algorithms concerning the expectation $y^{*}(k+1)$.

\subsection{Linearization algorithm}

\subsubsection{Linearization function}

First of all, following assumptions are set about the controller plant [17]:

Assumption 1. System (7) is observable, and controllable, that is, to the expected bounded system output signal $y^{*}(k+1)$, there exist a bounded feasible control input signal which drives the system output equal to the expected output.

Assumption 2. The partial derivative of $f(\ldots)$ in respect to control input $u(k)$ is continuous.

Assumption 3. The system applies for the generalized Lipschitz stabilization condition, that is

$$
\begin{aligned}
& \mid y\left(k_{1}+1\right)- y\left(k_{2}+1\right)|\leq b| u\left(k_{1}\right)-u\left(k_{2}\right) \mid, \\
& \forall k_{1} \neq k_{2} \cap k_{1}, k_{2} \geq 0 \cap u\left(k_{1}\right) \neq u\left(k_{2}\right)
\end{aligned}
$$

where $b$ is a positive constant which can be regarded as a limitation on the change of output in respect to the change of input. Assumption 3 applies to many common systems, such as temperature, pressure, or speed control in terms of energy: a bounded energy change of input leads to a bounded energy change of output.

To enclose all the nonlinear and time-varying information in a nonlinear system, the general NARMAX model (7) is adapted for a control-oriented strategy in a form like that described in Theorem 1.

Theorem 1. System (7) satisfying assumption 1, 2, and 3 can be rewritten as

$$
\Delta y(k+1)=\phi_{c}(k) \delta u(k), \forall \Delta u(k) \neq 0
$$

where $\Delta y(k+1)=y(k+1)-y(k)$ and $\Delta u(k)=u(k)-$ $u(k-1)$ are introduced to simplified the equation, and $\phi_{c}(k) \in R$, named as the PPD is a time-varying factor and bounded in respect to $k$.

Since $\phi_{c}$ records all the changes of output in respect to input and sampling instant, Theorem 1 indicates its ability to track the system's dynamics with the approximation of PPD.

\subsubsection{Estimation of PPD}

Obviously, it is hard to have a specific expression for a solution to the time-varying parameter $\phi_{c}(k)$, so a certain approximation algorithm is essential for the description of the system. Generally, the non-linear least square (LS) scheme is adopted as a simple and effective way to get a quick online solution. Unfortunately, such cost function is somewhat over sensitive to the temporary unreasonable results, which are probably caused by the unpredictable internal or external disturbances, failure of the encoder, etc. By including the disturbances into the calculation of the difference between the expected and actual output, the method may lead to an error in control output. Therefore, a weighting factor $\mu$ is introduced to punish the sudden change of $\phi_{c}(k)$. When substitute (9) into the advanced LS function, we have

$$
\begin{aligned}
J\left(\phi_{c}(k)\right)=\mid y(k)-y(k-1) & -\left.\phi_{c}(k) \Delta u(k-1)\right|^{2} \\
& +\mu\left|\phi_{c}(k)-\hat{\phi}_{c}(k-1)\right|^{2}
\end{aligned}
$$

where $\hat{\phi}_{c}(k)$ is the approximation of $\phi c(k)$ based on (10).

When the derivative of (10) in respect to $\phi_{c}(k)$ equals zero, an iterative approximation function is obtained

$$
\begin{aligned}
& \hat{\phi}_{c}(k)=\hat{\phi}_{c}(k-1)+ \\
& \quad \frac{\eta \Delta u(k-1)}{\mu+\Delta u(k-1)^{2}}\left(\Delta y(k)-\hat{\phi}_{c}(k-1) \Delta u(k-1)\right)
\end{aligned}
$$

where $\eta \in(0,1]$ is introduced as a weighting factor for a more flexible approximation rule to determine to what 
extend $\phi_{c}(k)$ is adjusted according to the I/O data. Also, the $\mu>0$ added to the denominator helps avoid a zero dividend in case that inputs remain the same during the sampling interval.

Additionally, a resetting rule described in (12) is supplemented for a greater ability to track nonlinear parameter

$$
\begin{aligned}
\hat{\phi}_{c}(k)=\hat{\phi}_{c}(1), \forall\left|\hat{\phi}_{c}(k)\right| & \leq \varepsilon \cap|\Delta u(k)| \leq \\
\varepsilon & \cap \operatorname{sgn}\left(\hat{\phi}_{c}(k)\right) \neq \operatorname{sgn}\left(\hat{\phi}_{c}(1)\right)
\end{aligned}
$$

where $\varepsilon$ is a sufficiently small positive number, and $\phi_{c}(1)$ is the self-defined initial value of $\phi_{c}(k)$.

\subsubsection{Controlling rules}

In discrete-time system, simply minimizing the onestep-ahead error between the control and actual output may lead to a large control input that is liable to run over the defined space which is probably harmful to the system. Take the USM as an example, frequency as low as $40 \mathrm{kHz}$ would destruct the inner material, such as the piezoelectric ceramic, and cause permanent damage to the motor. On the other hand, the weighted one-stepahead criterion often fails to eliminate the final steady state error. Therefore, a new cost function combining the two algorithms is considered to predict the control input

$$
J(u(k))=\left|y^{*}(k+1)-y(k+1)\right|^{2}+\lambda|u(k)-u(k-1)|^{2}
$$

where $\lambda$ is introduced as a weighting factor to restrict the variance of $u(k)$, and $y *(k+1)$ is the expecting output value. Similarly to (10), an iterative expression of $u(k)$ is obtained when the derivative of $J(u(k))$ in respect to $u(k)$ equals zero. Then we have

$$
u(k)=u(k-1)+\frac{\rho \phi_{c}(k)}{\lambda+\left|\phi_{c}(k)\right|^{2}}\left(y^{*}(k+1)-y(k)\right)
$$

where $\rho \in(0,1]$ is introduced as a penalty factor for a more flexible controlling rule.

Finally, the estimation algorithm (11), the resetting rules (12), and the controlling equation (14) constitute the whole MFAC scheme, and it can be summed as

$$
\begin{aligned}
\hat{\phi}_{c}(k)= & \hat{\phi}_{c}(k-1)+ \\
& \frac{\eta \Delta u(k-1)}{\mu+\Delta u(k-1)^{2}}\left(\Delta y(k)-\hat{\phi}_{c}(k-1) \Delta u(k-1)\right), \\
\hat{\phi}_{c}(k)= & \hat{\phi}_{c}(1), \forall\left|\hat{\phi}_{c}(k)\right| \leq \\
& \varepsilon \cap|\Delta u(k)| \leq \varepsilon \operatorname{sgn}\left(\hat{\phi}_{c}(k)\right) \neq \operatorname{sgn}\left(\hat{\phi}_{c}(1)\right), \\
u(k)= & u(k-1)+\frac{\rho \hat{\phi}_{c}(k)}{\left(\lambda+\left|\hat{\phi}_{c}(k)\right|^{2}\right)\left(y^{*}(k+1)-y(k)\right)} .
\end{aligned}
$$

Obviously, we would expect the greatest $\alpha$ when $\lambda=1$ and the smallest value when $\lambda=20$ in Fig. 6(b). Unfortunately, even the smallest value was nearly twice the expectation which was undesirable and may cause damage to the limited-input USM system in practical application. Referring to (15), the control input was largely affected by $\phi_{c}(k)$. So, to figure out the overshoot problem, the corresponding curves of $\phi$ were drawn in Fig. 6(c). The smallest change of $\phi$ was expected to appear in the curve of $\lambda=20$ when the initial value of $\phi$ were all set to be 1 , which agrees with the change of the overshoot. Thus, a proper initial value of $\phi$ would be a promising solution to address the overshoot problem.

On the other hand, since all the curves shared a similar rising time as shown in Fig. 7(a), we chose $\lambda=20$ as the ideal value for the current USM system. 

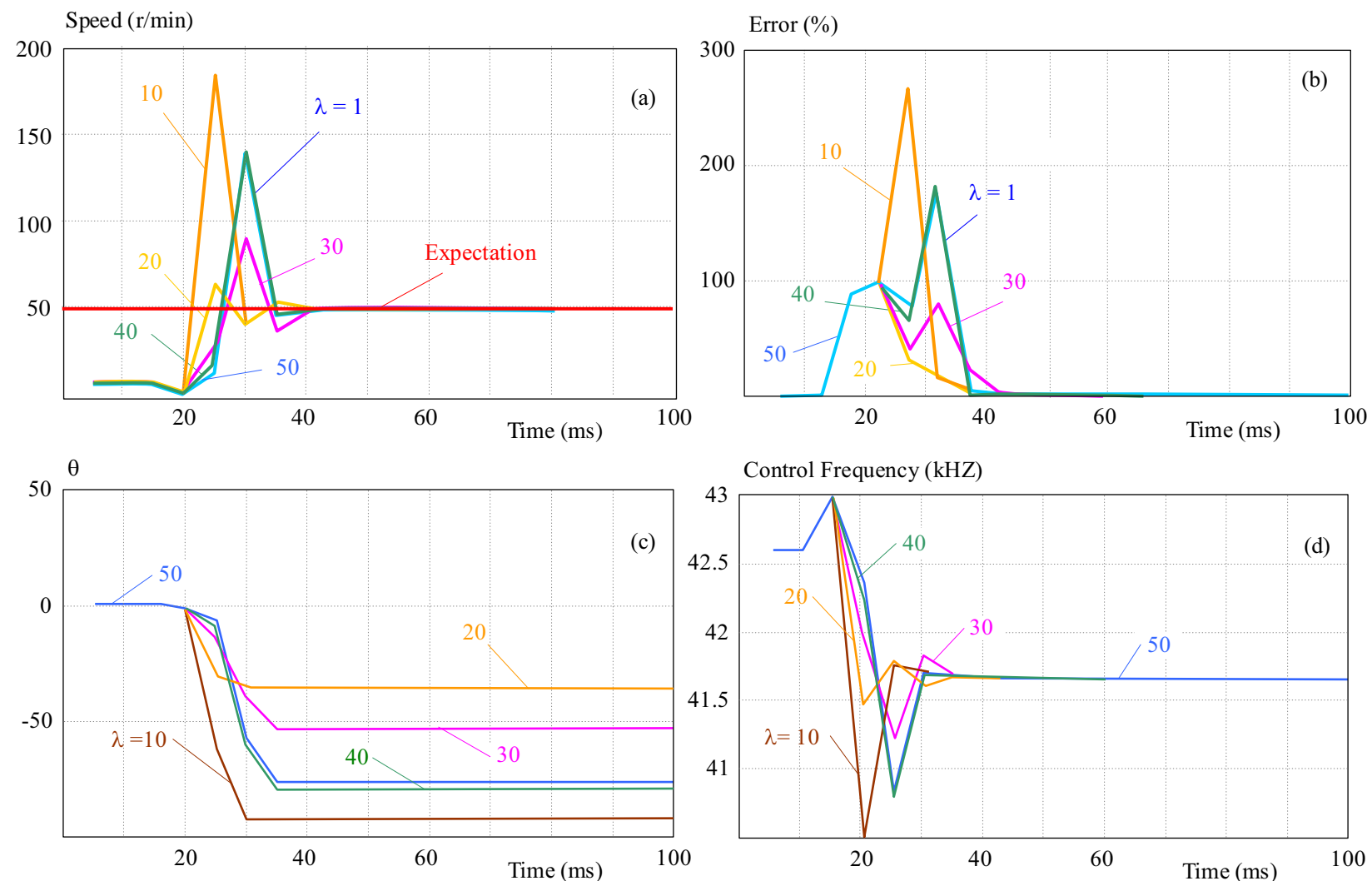

Fig. 6. The control results for constant speed under different $\lambda$
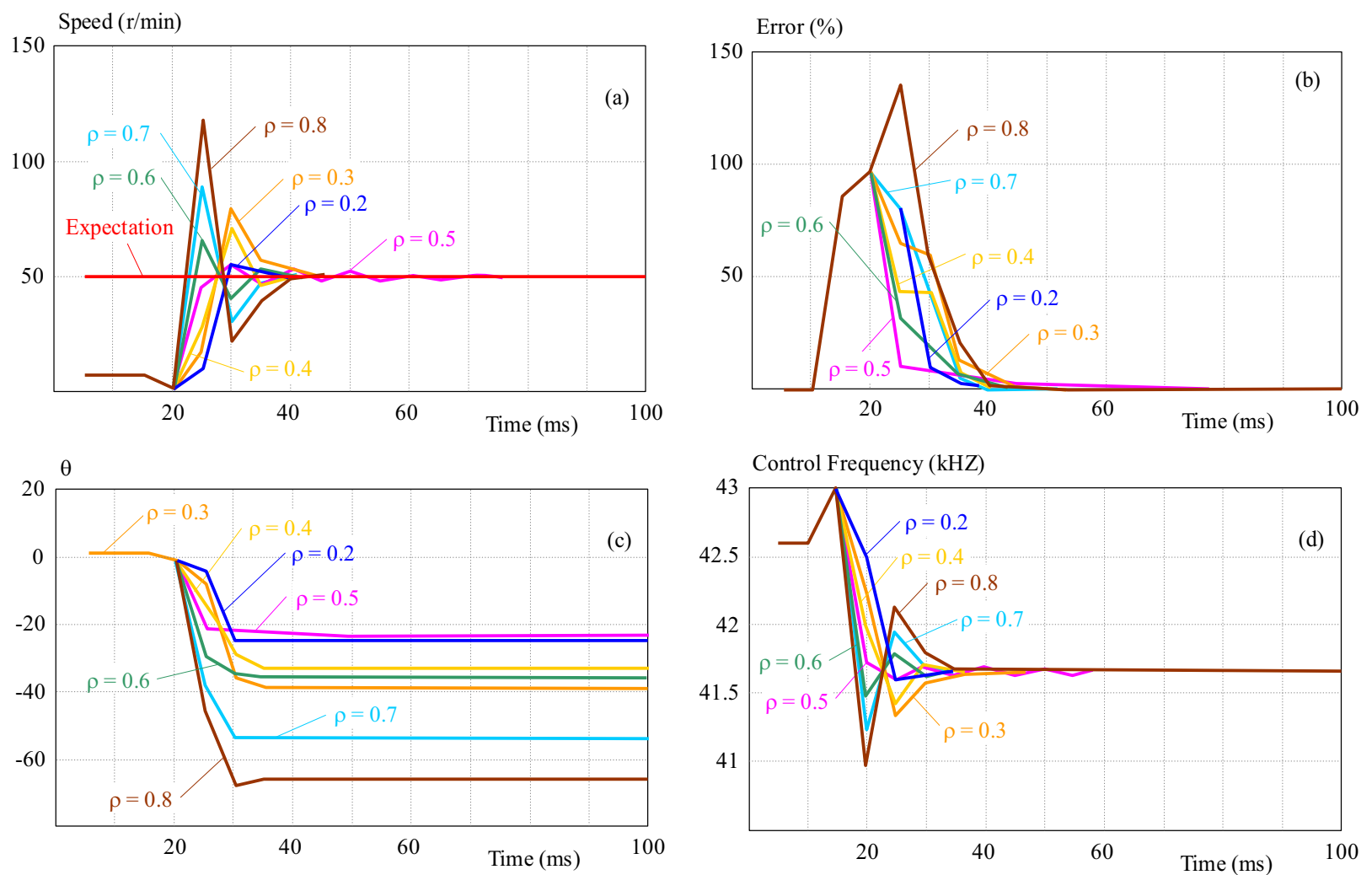

Fig. 7. The control results for constant speed under different $\rho$

The impact of $\rho$

When rewrite (14) as (17), $\rho$ can be regarded as the weight of the function and determine to what extend the control output is adjusted

$$
\Delta u(k)=\rho \frac{\phi_{c}(k)}{\lambda+\left|\phi_{c}(k)\right|^{2}}\left(y^{*}(k+1)-y(k)\right) .
$$



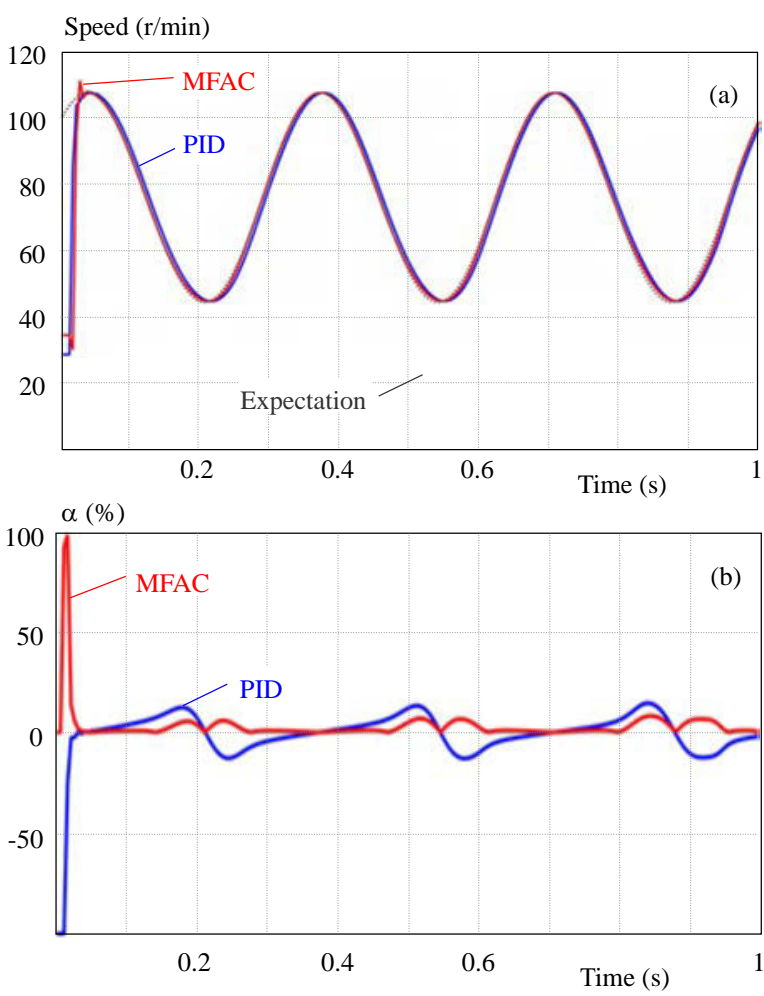

Fig. 8. Tracking results of MFAC and PID under the ideal sine wave

Theoretically, we would expect an opposite impact of $\rho$ in the numerator on the results against the $\lambda$ in the denominator. Figure 7 shows the impact of $\rho$ on the results when $\eta=\mu=1, \lambda=20$. The overshoot in respect to $\rho$ was neither monotonous as that presented in Fig. 8, which decreased as $\rho$ decreased from 0.8 and continued to increase as $\rho$ increased to 0.2 with the minimum appeared at $\rho=0.5$. Figure $7(\mathrm{~b})$ and (c) demonstrated the largest error rate at $\rho=0.8$ and the smallest change of $\phi$ at $\rho=0.5$ respectively, which agrees with the theoretical assumption. To make it a little different, the curves of $\rho=0.6$ enjoyed a shorter tuning time with a slightly greater value of the overshoot than that of $\rho=0.5$, which indicated another possible choice when real-time tracking is the main concern. In this way, 0.5 and 0.6 would both be the proper value for $\rho$.

In fact, since the relationship among $\lambda, \rho$, and $u(k)$ is nonlinear, the final value of $\lambda$ and $\rho$ would float around the above values due to their combined effect on the control input.

\section{Results}

To demonstrate the features of MFAC, the results of a control system based on MFAC is compared with that of the traditional PID method. All simulations were carried out with the same initial values and sample instant. According to the discussion in Section $4, \eta=\mu=1$, $\rho=0.5, \lambda=30$ were adjusted for MFAC. For the
PID method, satisfying results were obtained when $K_{p}=$ $0.001, K_{i}=2.5, K_{d}=0$.

\section{Comparison under the ideal wave}

The ideal periodic wave $y=40 \sin (6 \pi t+\pi / 4)+60$ was used to test the two methods. Figure 8 showed that MFAC is superior to PID with less delay in time and smaller error rate, while the overshoot was a little larger than that of PID which could probably owe to improper initial values. Fortunately, the value was still within the regulated range of control output, which was acceptable by the USM to avoid the damage to the controlled plant.

\section{Comparison under arbitrary wave with random noise}

An arbitrary wave described as $y=80+30 \operatorname{rand}()+$ $10 \operatorname{rand}()$ was introduced to present the advantages of MFAC over PID. A random signal generated by $\operatorname{rand}()$ function in Matlab was added to the constant speed to mimic the arbitrary expectation and inevitable noise. Figure 9 presented a much smaller error of MFAC than that of PID when tracking a nonlinear system with many uncertainties. This can be owed to the time-varying PPD $\phi$ included in the control rules which allows the output to be more adaptive.

In the end, it is believed that MFAC would operate better than the traditional PID control method with fewer factors to determine and is more robust to the change of expectation and disturbances.

\section{Conclusion}

In this paper, a novel model-free adaptive control (MFAC) scheme for ultrasonic motor in the presence of fully unknown model dynamics due to inertia uncertainties and external disturbances is first proposed. To test the efficiency of the proposed method, a speed-frequencytime model was established based on the practical experiments. Then, PSO was applied to identify the experimentbased model, whose parameters were studied for a deeper understanding of the nonlinearity of the operation system. Furthermore, the MFAC was presented in terms of its principles, algorithms and parameter selection. Finally, the ideal periodic system and system with random noise were used to demonstrate that the efficiency of MFAC is superior to that of PID. Thus, MFAC was proved to be a suitable method for controlling a highnonlinear system.

Considering the disadvantages of MFAC discovered in its comparison with PID, a larger overshoot could to be eliminate with a proper initial value of . On the other hand, the adaptive selection of parameters, a quicker convergence may be the future topics. Also, the proposed method will be tested on the benchmark for the final application verification. 

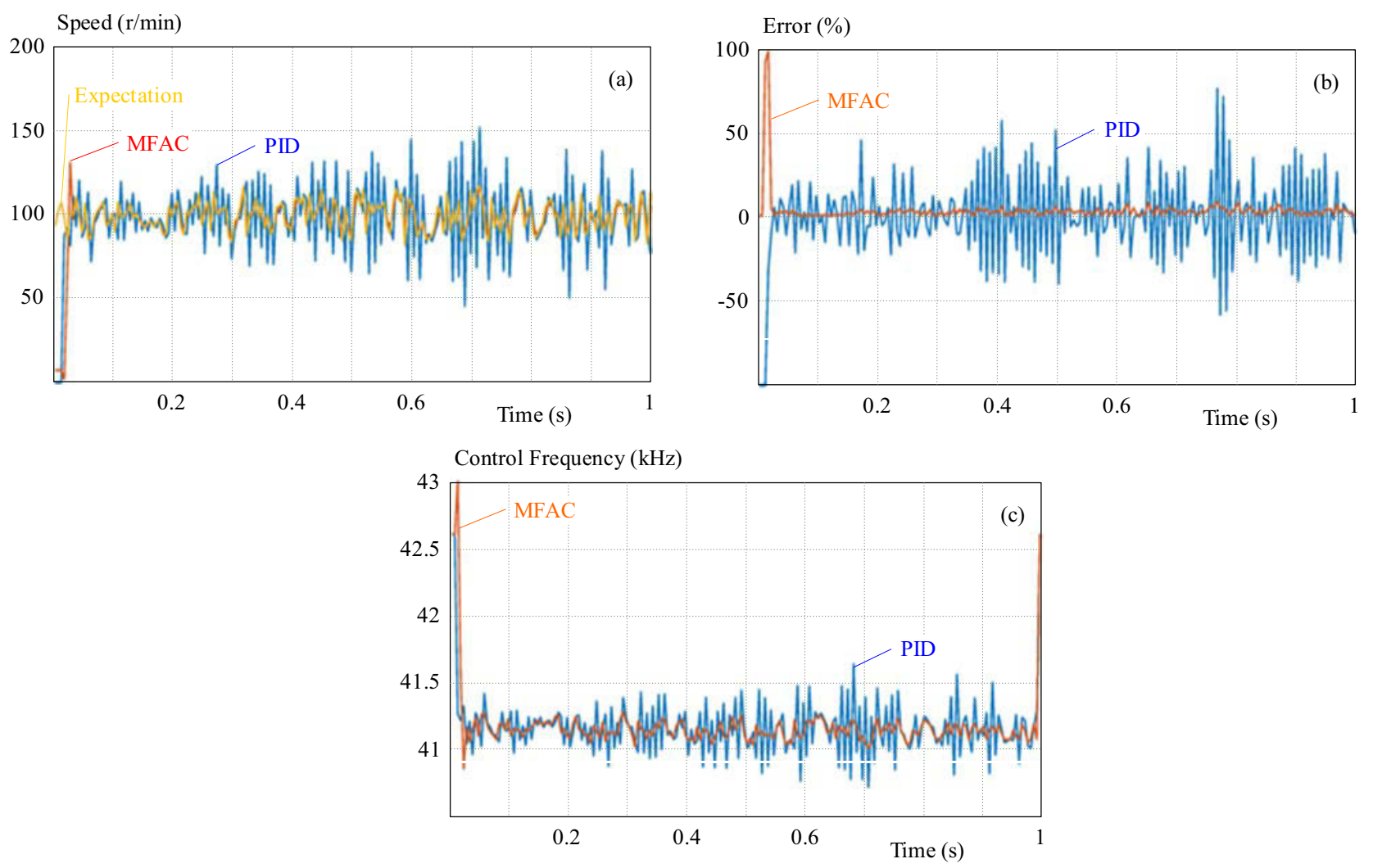

Fig. 9. Tracking results of MFAC and PID under arbitrary wave with random noise

\section{Acknowledgements}

This work was supported by the National Basic Research Program of China (973 Program, Grant No. 2015CB057503).

\section{REFERENCES}

[1] T. Senjyu, H. Miyazato and K. Uezato, "Quick and Precise Position Control of Ultrasonic Motors with Dual Mode Control", International Journal of Electronics, vol. 80, pp. 605-609, 1996.

$[2]$ T. Senjyu, H. Miyazato and K. Uezato, "Performance Comparison of PI and Adaptive Controller for Adjustable Speed Drives of Ultrasonic Motors", Industrial Technology Proceedings of the IEEE International Conference, 1994.

[3] T. Senjyu, H. Miyazato and K. Uezato, "Precise Speed Control of Ultrasonic Motors vvit h Repetitive Control", Industrial Automation and Control: Emerging Technologies, International IEEE/IAS Conference, 1995.

[4] T. Senjyu, H. Miyazato and K. Uezato, "Quick and Precise Position Control of Ultrasonic Motors with Two Control Input", Electric Machines \& Power Systems, vol. 25, pp. 767-781, 1997.

[5] F. J. Lin, "Fuzzy Adaptive Model-Following Position Control for Ultrasonic Motor", IEEE transactions on power electronics, vol. 12, no. 2, pp. 261-268, 1997.

[6] F. J. Lin, R. J. Wai and R. Y. Duan, "Neural-Network Controller for Parallel-Resonant Ultrasonic Motor Drive", IEEE transactions on control systems technology, vol. 7 no. 4, pp. 494-501, 1999.

[7] F. J. Lin, R. J. Wai and R. Y. Duan, "Fuzzy Neural Networks for Identification and Control of Ultrasonic Motor Drive with LLCC Resonant Technique", IEEE transactions on industrial electronics, vol. 46, no. 5, pp. 999-1011, 1999.
[8] F. J. Lin, R. J. Wai and C. M. Hong, "Recurrent Neural Network Control for LCC-Resonant Ultrasonic Motor Drive", IEEE transactions on ultrasonics, ferroelectrics, and frequency control, vol. 47 , no. 3, pp. 737-749, 2000.

[9] S. Mu and K. Tanaka, "Position Control of Ultrasonic Motor Using PID-IMC Combined with Neural Network based on Probability", International Journal of Applied Electromagnetics and Mechanics, 2013.

[10] S. Mu, K. Tanaka and S. Nakashima, "Intelligent PID Control for USM Using PSO Real-Time Environment", International Symposium on Computer, Consumer and Control, 2014.

[11] S. Mu, K. Tanaka, S. Nakashima and Alrijadjis, "Intelligent Control of Ultrasonic Motor Using PSO Type Neural Network", 14th ACIS International Conference on Software Engineering, Artificial Intelligence, Networking and Parallel/Distributed Computing, 2013.

[12] J. Z. Shi, B. Liu and Y. Zhang, "Study on Self-Tuning Pole Assignment Speed Control of an Ultrasonic Motor", ISA Transactions, vol. 50, pp. 581-587, 2011.

13] J. Z. Shi and B. Liu, "Optimum Efficiency Control of Traveling-Wave Ultrasonic Motor System", IEEE transactions on industrial electronics, vol. 58, no. 10, pp. 4822-4829, 2011.

[14] J. Z. Shi, F. F. Lv and B. Liu, "Self-Tuning Speed Control of Ultrasonic Motor Combined with Efficiency Optimization", International Journal of Control, Automation, and Systems, vol. 12, no. 1, pp. 93-101, 2014

[15] J. Z. Shi and B. Liu, "Non-Linear Generalized Predictive Control of Traveling-wave Ultrasonic Motor", Electric Power Components and Systems, vol. 40, pp. 1229-1245, 2012.

[16] J. Z. Shi, Y. Liu, J. T. Huang, M. Y. Xu, J. W. Zhang and L. Zhang, "Novel Intelligent PID Control of Traveling Wave Ultrasonic Motor", ISA Transactions, vol. 53, pp. 1670-1679, 2014.

17] Z. Hou and S. Jin, "The Model Free Adaptive Control - Theory and Application", Science Press, 2013. 
[18] J. Robinson and Y. Rahmat-Samii, "Particle Swarm Optimization Electromagnetics", IEEE transactions on antennas and propagation, vol. 52, no. 2, pp. 397-407, 2004.

[19] Y. Shi and R. C. Eberhart, "Parameter Selection Particle Swarm Optimization", International Conference on Evolutionary Programming, 1998.

[20] J. Kennedy and R. Eberhart, "Particle Swarm Optimization", IEEE international conference on neural networks proceedings, 1995.

[21] S. A. Billings, "Nonlinear System Identification: NARMAX Methods, the Time, Frequency, and Spatio-temporal Domains". Wiley, 2013.

Received 25 February 2017

Sisi Di received the BE degree in electrical engineering and automation from Nanjing University of Information Science and Technology, Jiangsu, China, in 2011. She is currently pursuing the $\mathrm{PhD}$ degree in instrument science and technology in Nanjing University of Aeronautics and Astronautics, Jiangsu, China. Her research interest includes the control of ultrasonic motors, model-free control method and data-driven control of nonlinear systems. She has published 3 patents and several articles in international conferences, including the International Workshop on Piezoelectric Materials and Applications in Actuators (IWPMA) 2014.

Huafeng Li, received the $\mathrm{PhD}$ degree in Electrical Engineering from Huazhong University of Science and Technology, Hubei, China, in 2002. After that, he became a post-doctoral researcher and then a professor in Nanjing University of Aeronautics and Astronautics (NUAA). He is now a professor of NUAA, specialist in electrical engineering. is main research fields include ultrasonic motor and its applications, drive and control technology of ultrasonic motor, MFC, IPMC smart materials and structures, and precise driving technology. In 2013, an ultrasonic motor developed by his group was successfully used in Chang'e 3 rover, which was the first application of ultrasonic motor in aerospace in China. He hosted three projects supported by National Science Foundation of China, won 2013 National Award for Technological Invention 2nd Prize and 2012 Ministry of Education Award for Technological Invention 1st Prize (the 5th accomplisher). Meanwhile, he has published more than 70 articles and obtained 10 patents. 